\title{
SNR Estimation in Time-Varying Fading Channels
}

\author{
Ami Wiesel, Student Member, IEEE, Jason Goldberg, Senior Member, IEEE, and Hagit Messer-Yaron, Fellow, IEEE
}

\begin{abstract}
Signal-to-noise ratio (SNR) estimation is considered for phase-shift keying communication systems in time-varying fading channels. Both data-aided (DA) estimation and nondata-aided (NDA) estimation are addressed. The time-varying fading channel is modeled as a polynomial-in-time. Inherent estimation accuracy limitations are examined via the Cramer-Rao lower bound, where it is shown that the effect of the channel's time variation on SNR estimation is negligible. A novel maximum-likelihood (ML) SNR estimator is derived for the time-varying channel model. In DA scenarios, where the estimator has a simple closed-form solution, the exact performance is evaluated both with correct and incorrect (i.e., mismatched) polynomial order. In NDA estimation, the unknown data symbols are modeled as random, and the marginal likelihood is used. The expectation-maximization algorithm is proposed to iteratively maximize this likelihood function. Simulation results show that the resulting estimator offers statistical efficiency over a wider range of scenarios than previously published methods.
\end{abstract}

Index Terms-Cramer-Rao bound (CRB), expectation-maximization (EM), maximum-likelihood (ML) estimation, signal-tonoise ratio (SNR).

\section{INTRODUCTION}

$\mathbf{M}$ ODERN wireless communication systems often require knowledge of the signal-to-noise ratio (SNR) at the receiver. For example, SNR estimates are typically employed in power control, mobile assisted handoff, and adaptive modulation schemes, as well as soft decoding procedures [1], [2]. The rapid development of these applications in the last decade has led to an intense search for accurate and low-complexity SNR estimators.

The problem of SNR estimation may be considered for dataaided (DA) scenarios, where known transmitted data is used to facilitate the estimation process, and for nondata-aided (NDA) scenarios, since the periodic transmission of known data limits system throughput. The basic problem was first introduced in the 1960s by [3] and [4]. However, decreasing hardware cost and increasing demands for pushing system performance to the

Paper approved by R. A. Kennedy, the Editor for Data Communications Modulation and Signal Design of the IEEE Communications Society. Manuscript received May 11, 2003; revised October 28, 2003 and April 23, 2004. This paper was presented in part at the IEEE International Conference on Communications, New York, NY, 2002, and in part at the IEEE International Conference on Acoustics, Speech, and Signal Processing, Orlando, FL, 2002.

A. Wiesel was with the Department of Electrical Engineering-Systems, Tel Aviv University, Ramat Aviv 69978, Israel. He is now with the Department of Electrical Engineering, Technion-Israel Institute of Technology, Haifa 32000, Israel (e-mail: amiw@tx.technion.ac.il).

J. Goldberg was with the Department of Electrical Engineering-Systems, Tel Aviv University, Ramat Aviv 69978, Israel. He is now with Global Locate Inc., San Jose, CA 95124 USA.

H. Messer-Yaron is with the Department of Electrical Engineering-Systems, Tel Aviv University, Ramat Aviv 69978, Israel (e-mail: messer@eng.tau.ac.il).

Digital Object Identifier 10.1109/TCOMM.2006.873995 achievable limits has caused the topic to rise to prominence once again.

Over the last decade, attention has focused on SNR estimation for constant, frequency-flat channels [5], [6], as well as for constant, frequency-selective channels [7], [8]. Various techniques have been proposed including the maximum-likelihood (ML) estimation method, the decision-directed (DD) method, and the method of moments (MM). Moreover, the inherent accuracy limitations associated with SNR estimation in a static channel with no intersymbol interference (ISI) have been investigated in [6] and [9]. This paper focuses on two main aspects of the problem: NDA estimation, and estimation in frequency-flat, time-varying fading channels.

NDA estimation: The two standard NDA SNR estimators are the DD estimator and the MM estimator and their variants. None of these estimators attain the Cramer-Rao bound (CRB) over a wide range of SNRs. In this paper, the problem of NDA estimation is confronted using the assumption of independent and identically distributed (i.i.d.) symbols and the associated marginal likelihood. This assumption allows for new estimation algorithms and performance bounds. In particular, we extend the work of [9], which first derived the CRB associated with the marginal likelihood. Finally, we propose a novel ML estimator based on the marginal likelihood. There is no closed-form solution for this ML estimate. As an alternative to a potentially costly exhaustive multidimensional search, we propose the use of the expectation-maximization (EM) algorithm [10], [11].

Estimation in time-varying fading channels: All of the previous estimators are based on the assumption that the channel is constant throughout the observation period. In many applications requiring SNR estimation (e.g., mobile communication), this assumption is not valid [12]. In particular, in NDA estimation, the channel is likely to be time-varying, since a relatively long observation period is required for satisfactory performance. Following [11] and [13], a time-varying, complex, fading channel model is introduced. ${ }^{1}$ The channel is approximated as a polynomial-in-time with a few unknown coefficients. A new CRB is derived where the received data distribution is that associated with the new channel model. The main results of this bound are that the effect of the channel's time variation on SNR estimation is negligible, and that there is no inherent penalty in asymptotic performance due to overestimating the order of this variation. These results motivate the design of new SNR estimators for the time-varying channel model. Hence, a novel DA and NDA ML estimator is derived. In DA scenarios, where the estimator has a simple closed-form solution, exact performance is evaluated both with correct and incorrect (i.e., mismatched) polynomial order. In NDA scenarios,

\footnotetext{
${ }^{1}$ The extension to frequency-selective channels is, at least conceptually, straightforward.
} 
an EM-based estimator is suggested, which is very similar to the approach taken in [11] in the context of joint time-varying channel estimation and data detection.

\section{PROBLEM Formulation}

Consider the reception of a wireless communications phaseshift keying (PSK)-type signal which passes through a timevarying, multipath channel. When the signal is of sufficiently narrow bandwidth, the channel frequency response may be considered flat over the frequency band of interest. In the time domain, this results in a time-varying, multiplicative channel gain. Assuming an ideal receiver with perfect synchronization, the symbol-spaced output of the receiver's matched filter can be written as

$$
y_{n}=a_{n} h_{n}+w_{n}, \quad n=1, \ldots, N
$$

where at the time index $n, y_{n}$ is the received sample, $a_{n}$ is the transmitted data symbol, $h_{n}$ is the time-varying channel gain, and $w_{n}$ is a realization of a zero-mean, white, complex normal process of variance $\sigma^{2}$. These received samples can be conveniently represented in $N \times 1$ column vector form

$$
\mathbf{y}=\mathbf{A h}+\mathbf{w}
$$

where $\mathbf{y}=\left[y_{1} \cdots y_{N}\right]^{T}, \mathbf{h}=\left[h_{1} \cdots h_{N}\right]^{T}, \mathbf{w}=\left[w_{1} \cdots w_{N}\right]^{T}$, and $[\cdot]^{T}$ denotes the transpose operator. The $N \times N$ matrix $\mathbf{A}$ is a diagonal matrix with $\mathbf{A}_{n n}=a_{n}$. We will deal with the binary PSK (BPSK) constellation, i.e., $a_{n} \in\{ \pm 1\}$, and the quadrature PSK (QPSK) constellation, i.e., $a_{n} \in\{ \pm \sqrt{(1 / 2)} \pm j \sqrt{(1 / 2)}\}$.

Clarke's flat-fading channel model will be used, wherein $h_{n}$ is modeled as a realization of a zero-mean, complex normal random process with the correlation function (e.g., [12]) $\mathrm{E}\left[h_{n} h_{n-l}^{*}\right]=\sigma_{h}^{2} J_{0}\left(2 \pi\left(f_{d} / f_{s}\right) l\right)$, where $\mathrm{E}[\cdot]$ denotes the expectation operator, $[\cdot]^{*}$ denotes the conjugate operator, $\sigma_{h}^{2}$ is the channel variance, $f_{d}$ is the maximum Doppler frequency, $f_{s}$ is the sampling rate, and $J_{0}(\cdot)$ is the zeroth-order Bessel function.

Generally, the complex channel $h_{n}$ is completely unknown to the receiver. However, in some applications, it is reasonable to assume that the receiver has a separate phase-synchronization mechanism, such that the phase of the channel is known and is compensated for [14]. In such applications, $h_{n}$ denotes a timevarying, real channel.

In most applications, $N$ is such that $\left(f_{d} / f_{s}\right) N \ll 1$, i.e., the channel is highly correlated and almost "constant" during the observation interval. In practice, the channel is traditionally modeled as constant, i.e., $h_{n}=h_{0}$. However, as will be seen in the following, even small variations from this channel model can dramatically degrade the performance of traditional, constant channel, SNR estimators.

A more appropriate time-varying channel model is the polynomial-in-time model [11], [13]. The slowly time-varying process $h_{n}$ is, by definition, bandlimited. Therefore, it can be expanded as an $L_{C}$-order polynomial-in-time, using Taylor's theorem

$$
h_{n}=\sum_{m=0}^{L_{C}-1} c_{m} t_{n}^{m}+R_{L_{C}}(n)
$$

where $c_{m}$ is the $m$ th coefficient, $t_{n}$ is the time index of the $n$th sample, and $R_{L_{C}}(n)$ is the remainder of the Taylor series. In real channels, $c_{m}$ is a real coefficient. Otherwise, in complex channels, each coefficient is actually two real coefficients, $c_{m}=\alpha_{m}+j \beta_{m}$. The mean-squared value of $R_{L_{C}}(n)$ approaches zero as $L_{C} \rightarrow \infty$ or as $\left(f_{d} / f_{s}\right) N \rightarrow 0$ [13]. Therefore, for $L_{C}$ sufficiently high, the channel $\mathbf{h}$ can be accurately approximated as $\mathbf{h} \approx \mathbf{T}_{L_{C}} \mathbf{c}$, where $\mathbf{T}_{L_{C}}=\left[\mathbf{t}_{1} \cdots \mathbf{t}_{N}\right]^{T}, \mathbf{t}_{n}=$ $\left[t_{n}^{0} \cdots t_{n}^{L_{C}-1}\right]^{T}, \boldsymbol{\alpha}=\left[\alpha_{0} \cdots \alpha_{L_{C}-1}\right]^{T}, \boldsymbol{\beta}=\left[\beta_{0} \cdots \beta_{L_{C}-1}\right]^{T}$, and $\mathbf{c}=\left[c_{0} \cdots c_{L_{C}-1}\right]^{T}$. For ease of notation, we sometimes omit the subscript in $\mathbf{T}_{L_{C}}$ and simply refer to $\mathbf{T}$.

Different problem scenarios give rise to different probability density function (PDF) models for the received vector. Assuming the polynomial coefficients are deterministic unknown parameters, the PDF of each received sample is either complex normal (DA) or a mixture of complex normals (NDA). For example, in the case of BPSK transmission, the PDFs of each sample are

$$
\begin{aligned}
p_{\mathrm{DA}}\left(y_{n} ; \mathbf{c}, \sigma^{2}, a_{n}\right) & =\frac{1}{\pi \sigma^{2}} \exp \left(-\frac{\left|y_{n}-a_{n} \mathbf{t}_{n}^{T} \mathbf{c}\right|^{2}}{\sigma^{2}}\right) \\
p_{\mathrm{NDA}}\left(y_{n} ; \mathbf{c}, \sigma^{2}\right) & =\frac{1}{2} \sum_{a_{n} \in\{ \pm 1\}} p_{\mathrm{DA}}\left(y_{n} ; \mathbf{c}, \sigma^{2}, a_{n}\right) .
\end{aligned}
$$

Due to the received samples' independence, the overall received vector PDF is the product of the $N$ received samples' PDFs.

While various definitions of SNR are possible, in this paper, the term SNR refers to a "locally averaged SNR," i.e., the SNR over a specific channel realization

$$
\rho=\frac{\sum_{n=1}^{N}\left|h_{n}\right|^{2}}{N \sigma^{2}}=\frac{\mathbf{c}^{H} \mathbf{T}^{T} \mathbf{T} \mathbf{c}}{N \sigma^{2}} .
$$

The SNR estimation problem may be stated as: Given the known symbols $\mathbf{a}_{\mathrm{DA}}$ and the observation vector $\mathbf{y}$ along with its statistical model, estimate the SNR. We will also be interested in the SNR (in decibels) to be denoted as $\rho^{(\mathrm{dB})}=10 \log \rho$.

\section{PERFORMANCE LimitATIONS}

The inherent performance limitations on SNR estimation are investigated using the CRB [10]. The importance of the CRB lies in the fact that, as the number of samples increases, it may be attained by the ML estimator. Therefore, we will use this bound in order to evaluate the asymptotic estimation performance. In addition, this analysis will give insight as to the effect of the channel's time variation and unknown phase on SNR estimation. 
The CRB for SNR estimation is

$$
\mathrm{CRB}(\rho) \equiv \frac{\partial \rho^{T}}{\partial \boldsymbol{\theta}} \mathbf{J}_{\boldsymbol{\theta} \boldsymbol{\theta}^{T}}^{-1} \frac{\partial \rho}{\partial \boldsymbol{\theta}}
$$

where the SNR $\rho(\theta)$ is a function of a vector of unknown parameters $\theta=\left[\mathbf{c}^{T}, \sigma^{2}\right]^{T}$ in real channels, or $\boldsymbol{\theta}=\left[\boldsymbol{\alpha}^{T}, \boldsymbol{\beta}^{T}, \sigma^{2}\right]^{T}$ in complex channels, and $\mathbf{J}_{\boldsymbol{\theta} \boldsymbol{\theta}^{T}}$ is the Fisher information matrix (FIM). Likewise, the $\mathrm{CRB}$ for $\rho^{\mathrm{dB}}$ is $\mathrm{CRB}^{\mathrm{dB}}(\rho)=100$. $\operatorname{CRB}(\rho) / \rho^{2} \ln ^{2}(10)$.

The derivation of the CRB involves tedious algebraic manipulations. These mainly consist of the derivations of the FIM elements which are provided in Appendix I, and the application of (6). We now give the major final results.

\section{A. DA Scenarios}

The CRB for DA SNR estimation is derived using (6) with the associated FIM elements in Appendix I. The result has a simple closed form

$$
\operatorname{CRB}_{\mathrm{DA}}(\rho)=\frac{\rho}{N}[2+\rho] .
$$

This bound has been previously presented for the special case of constant $\left(L_{C}=1\right)$, real channels with PSK modulation in [5]..$^{2}$ However, straightforward application of the FIM elements in (6) result in bound (7) for all of the possible DA problem formulations, including BPSK and QPSK constellations, as well as for complex channels (i.e., there is no degradation in asymptotic performance due to unknown phase). Moreover, the DA bound for time-varying channels $\left(L_{C}>1\right)$ coincides with (7), as well, for all $L_{C}$. This is quite surprising, as it means that the asymptotic performance does not depend on the number of unknown channel parameters. Therefore, for the same SNR, the bound in a constant channel with a few unknown parameters is identical to the bound in a time-varying channel with many unknown parameters. This implies that there is no inherent penalty in asymptotic performance incurred by overestimating $L_{C}$. Finally, as will be explained in the following, this bound also holds for NDA estimation in high SNR. This is intuitively reasonable, since high SNR implies an ability to perfectly estimate the transmitted symbols, effectively yielding a DA-type problem.

\section{B. NDA, Constant Channel Scenarios}

The CRB for the NDA, constant channel scenario can be derived through (6) with the FIM elements associated with the $L_{C}=1$ channel [9]

$$
\operatorname{CRB}_{\text {NDA }}(\rho)= \begin{cases}\frac{\rho}{N} \frac{2+\rho f(2 \rho)}{f(2 \rho)+2 \rho f(2 \rho)-2 \rho}, & \text { BPSK } \\ \frac{\rho}{N} \frac{2+\rho f(\rho)}{f(\rho)+2 \rho f(\rho)-2 \rho}, & \text { QPSK }\end{cases}
$$

${ }^{2}$ In [5], the bound for BPSK constellations had an additional factor of two, compared with (7). This stems from the real-noise model used in that work.

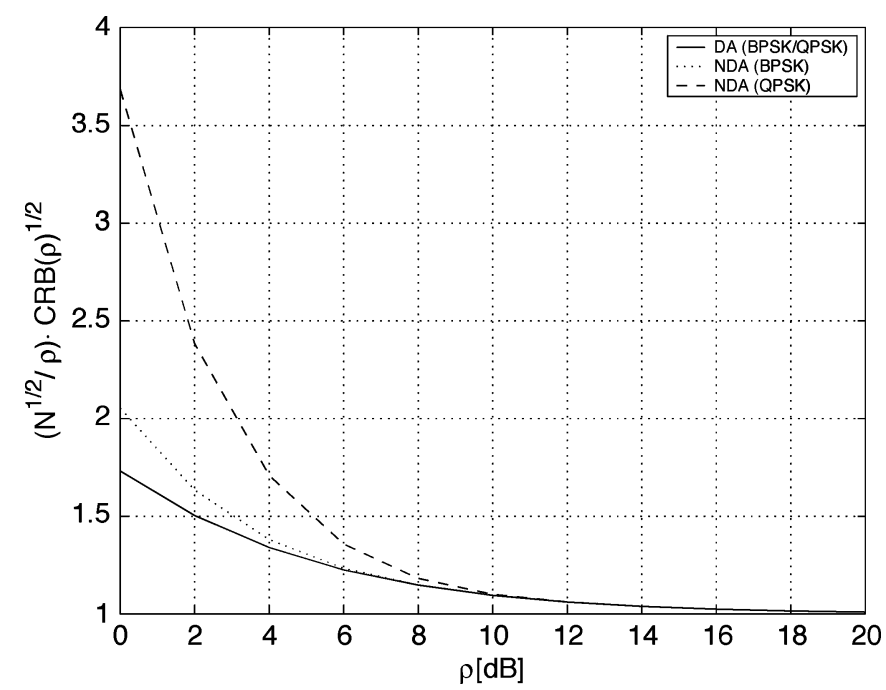

Fig. 1. Normalized root CRB for constant, real, and complex channels, $L_{C}=1$.

where

$$
f(\rho)=1-\frac{e^{-\frac{\rho}{2}}}{\sqrt{2 \pi}} \int_{-\infty}^{\infty} \frac{z^{2} e^{-\frac{1}{2} z^{2}}}{\cosh (\sqrt{\rho} z)} d z
$$

is a measure for the "information" each sample carries about its SNR. This function is monotonically increasing with its SNR, eventually converging to one when the BPSK symbol is known a priori, or can be estimated without error, i.e., infinite SNR (for proof, see Appendix II). For convenience, a finite series approximation of this integral, which eliminates the need for numerical integration, is provided in [15].

Examining the constant channel bounds in Fig. 1 reveals that generally, the lack of DA symbols degrades the performance, but at high SNR, this degradation is negligible. Moreover, the choice of symbol constellation results in different NDA bounds. This is very intuitive, as it is more difficult to decide on QPSK symbols than it is to decide on BPSK symbols (assuming the same symbol power).

While (8) appeared previously in [9], it was only derived for the special case of real channels. Using the complex FIM elements in Appendix I, and a cumbersome $3 \times 3$ matrix inversion, it is possible to show that this bound holds for complex channels, as well, i.e., asymptotically, unknown phase does not degrade the performance of SNR estimation in constant channels.

\section{NDA, Time-Varying Channel Scenarios}

A closed form for the general CRB in time-varying channels is not available. Numerical evaluation of it in different scenarios led to the following observations. As previously stated, the DA bound does not change when the channel becomes time-varying. This is not true for the NDA bound. Because of the time variation, some received samples carry more "information" than others. These samples are those with the higher channel gains, for which a reliable estimate of $a_{n}$ can be more easily obtained. In DA estimation, $a_{n}$ are known, and therefore, all the received samples carry the same "information." Generally, time variation 
can improve or degrade performance. However, numerical evaluation of the bound indicates that the effect of time variation is not significant (the peak change is around $\pm 5 \%$ ).

\section{SNR ESTIMATORS}

In this section, we will give a brief review of previously reported SNR estimators. Next, we will propose an improved estimator based on the polynomial-in-time model.

\section{A. Previous Estimators}

In [3] and [4], the DA ML estimator was first derived for constant channels. It may be written as

$$
\widehat{\rho}_{\mathrm{ML}}=\frac{\mathbf{y}^{H} \mathbf{P y}}{\mathbf{y}^{H} \mathbf{P}{ }^{\perp} \mathbf{y}}, \quad \mathbf{P} \equiv \frac{\mathbf{a a}^{H}}{N}, \quad \mathbf{P}^{\perp} \equiv \mathbf{I}-\mathbf{P}
$$

where $\mathbf{P}$ and $\mathbf{P}^{\perp}$ are projection matrices projecting onto the rank-one "signal-plus-noise" and rank- $N-1$ "noise" subspaces, respectively, and a is a length- $N$ vector with the symbols $a_{n}$. One of the first NDA SNR estimators is the intuitive, DD-based ML estimator of [3] and [4]. This estimator first decides on the symbols. For example, the BPSK symbol estimates for the constant, real channel are $\widehat{a}_{n}=\operatorname{sign}\left(y_{n}\right)$. It then uses these decisions in the DA ML estimator as if they were the correct transmitted symbols.

Another well-known NDA estimator for constant channels is based on estimates of the second and fourth moments of the data, and will be referred to as the MM estimator, e.g., [5]

$$
\widehat{\rho}_{\mathrm{MM}}=\frac{\sqrt{2 \hat{m}_{2}^{2}-\widehat{m}_{4}}}{\widehat{m}_{2}-\sqrt{2 \hat{m}_{2}^{2}-\widehat{m}_{4}}}
$$

where $\hat{m}_{2}=(1 / N) \sum_{n=1}^{N}\left|y_{n}\right|^{2}$ and $\hat{m}_{4}=$ $(1 / N) \sum_{n=1}^{N}\left|y_{n}\right|^{4}$. Use of even order moments gives rise to an estimator which has the attractive property of not requiring explicit symbol decisions. Simulation results in Section IV, as well as [5], indicate that this estimator can outperform the DD estimator at low SNR for sufficiently long data records. However, at high SNR, when the decisions of the DD estimator are highly accurate, the performance of the MM estimator is generally worse.

As will be seen in Section IV, all of these estimators perform well when used in a constant channel model. However, even small deviations from this model can dramatically degrade their performance.

\section{B. A Novel ML Estimator}

In this section, we introduce an estimator specifically designed for DA and NDA SNR estimation in the complex polynomial-in-time, time-varying fading-channel model. The derivation for the real channel is very similar, and is, therefore, omitted.

The novel estimator is based on the ML estimator associated with the normal mixture NDA PDF. There is no closed-form ML solution for such estimators. We therefore propose the use of the EM algorithm to iteratively find the parameter estimates which maximize the likelihood function. ${ }^{3}$

The EM algorithm is a general iterative method for finding at least a local maximum of a likelihood function given "incomplete data" [10]. In our context, the "incomplete data" is the received data vector and the known symbols ( $\left.\mathbf{a}_{\mathrm{DA}}\right)$, whereas the "complete" data includes the unknown symbols ( $\mathbf{a}_{\mathrm{NDA}}$ ), as well. Define the "complete" data log-likelihood function

$$
L\left(\mathbf{y} ; \theta, \mathbf{a}_{\mathrm{DA}}, \mathbf{a}_{\mathrm{NDA}}\right)=C-N \log \sigma^{2}-\sum_{n=1}^{N} \frac{\left|y_{n}-a_{n} h_{n}\right|^{2}}{\sigma^{2}}
$$

where $C$ is a constant. Observe that since $\mathbf{a}_{\mathrm{NDA}}$ are random, this function is a random variable. The EM algorithm is iterative. At the $i$ th iteration, it first finds the Expectation of this random function with respect to the missing data (here $\mathbf{a}_{\mathrm{NDA}}$ ), given the incomplete data and the current parameter estimates. Next, the parameter vector which Maximizes this expectation is found

$$
\begin{aligned}
Q\left(\widetilde{\theta} ; \widehat{\theta}_{i-1}\right) & =E\left[L\left(\mathbf{y} ; \widetilde{\theta}, \mathbf{a}_{\mathrm{DA}}, \mathbf{a}_{\mathrm{NDA}}\right) \mid \mathbf{y}, \mathbf{a}_{\mathrm{DA}}, \widehat{\theta}_{i-1}\right] \\
\widehat{\theta}_{i} & =\arg \max _{\widetilde{\theta}} Q\left(\widetilde{\theta} ; \widehat{\theta}_{i-1}\right) .
\end{aligned}
$$

Straightforward differentiation of this expectation yields the parameters which minimize it

$$
\begin{aligned}
\widehat{\mathbf{c}}_{i} & =\left(\mathbf{T}_{L_{E}}^{T} \mathbf{T}_{L_{E}}\right)^{-1} \mathbf{T}_{L_{E}}^{T} \widehat{\mathbf{A}}_{i}^{H} \mathbf{y} \\
\widehat{\sigma}^{2} & =\frac{\mathbf{y}^{H} \mathbf{y}-\widehat{\mathbf{c}}_{i}^{H} \mathbf{T}_{L_{E}}^{T} \mathbf{T}_{L_{E}} \widehat{\mathbf{c}}_{i}}{N}
\end{aligned}
$$

where $\mathbf{T}_{L_{E}}$ and $\widehat{\mathbf{c}}_{i}$ correspond to an $L_{E}$ polynomial order channel model, and $\widehat{\mathbf{A}}_{i}$ is a diagonal matrix with the following diagonal elements:

$$
\left(\widehat{\mathbf{A}}_{i}\right)_{n n}= \begin{cases}a_{n}, & \text { DA samples } \\ E\left[a_{n} \mid y_{n}, \widehat{\theta}_{i-1}\right], & \text { NDA samples. }\end{cases}
$$

In general, the NDA diagonal elements are the expected mean value of the unknown symbols. For example, these soft decisions on the symbols in BPSK transmission can be derived as $E\left[a_{n} \mid y_{n}, \widehat{\mathbf{t}}_{i-1}\right]=\tanh \left[\left(2 \Re\left\{y_{n} \widehat{h}_{n, i-1}^{*}\right\}\right) / \widehat{\sigma}_{i-1}^{2}\right]$, where $\tanh (\cdot)$ denotes the hyperbolic tangent, and $\hat{h}_{n, i-1}=\mathbf{t}_{n}^{T} \widehat{\mathbf{c}}_{i-1}$. Finally, the estimator for the SNR at each iteration is defined as

$$
\widehat{\rho}_{i}=\frac{\mathbf{y}^{H} \mathbf{P}_{i} \mathbf{y}}{\mathbf{y}^{H} \mathbf{P}_{i}^{\perp} \mathbf{y}}
$$

where $\mathbf{P}_{i}=\widehat{\mathbf{A}}_{i} \mathbf{T}_{L_{E}}\left(\mathbf{T}_{L_{E}}^{T} \mathbf{T}_{L_{E}}\right)^{-1} \mathbf{T}_{L_{E}}^{T} \widehat{\mathbf{A}}_{i}^{H}$ and $\mathbf{P}_{i}^{\perp}=\mathbf{I}-\mathbf{P}_{i}$ are projection matrices projecting onto the "signal-plus-noise" and "noise" subspaces.

An important issue in any iterative algorithm is the selection of initial conditions. In real channels, the choice of initial conditions was not found to be crucial for convergence. Hence, an intuitive first iteration can be choosing infinite SNR, i.e., $\hat{\mathbf{c}}_{0}=[1, \cdots, 1]^{T}$ and $\hat{\sigma}_{0}=0^{2}$. Note that at high SNR, the sign

${ }^{3}$ It should be mentioned that this approach is similar to the one presented in [11], in the context of joint time-varying channel estimation and data detection. 
is a good approximation for the hyperbolic tangent, and therefore, the first iteration is actually the well-known DD estimator. Simulations show that using these initial conditions and five iterations leads to satisfactory performance.

On the other hand, in complex channels, the likelihood was found to have many local maxima. Therefore, using infinite SNR alone does not provide satisfactory initial conditions, and an initial phase estimate is required. For example, a simple phase estimate in BPSK can be [14] $\widehat{\operatorname{angle}}(h)=(1 / 2) \operatorname{angle}\left((1 / N) \sum_{n=1}^{N} y_{n}^{2}\right)$. In highly time-varying complex channels, finding good initial conditions is more difficult.

A known method for improving estimator performance is calculating and removing bias [5]. In the high-SNR NDA case, the matrix $\widehat{\mathbf{A}}_{i}$ is approximately equal to the matrix $\mathbf{A}$. In this case, as well as the DA case, it is easy to verify that the estimator is a scaled noncentral $F$ distributed random variable [16]

$$
\frac{N-L_{E}}{L_{E}} \widehat{\rho} \sim \mathcal{F}_{2 L_{E}, 2 N-2 L_{E}}(2 N \rho)
$$

where $\mathcal{F}_{\nu_{1}, \nu_{2}}(\lambda)$ denotes the noncentral $F$ distribution with degrees of freedom (DOFs) $\nu_{1}$ and $\nu_{2}$, and with noncentrality parameter $\lambda$. The moments of such random variables are well known in the literature [16]. Using the first moment, an unbiased (UB) estimator (i.e., $\mathrm{E}\left[\widehat{\rho}_{\mathrm{UB}}\right]=\rho$ ) can be derived

$$
\widehat{\rho}_{\mathrm{UB}}=\frac{N-L_{E}-1}{N} \widehat{\rho}-\frac{L_{E}}{N} .
$$

However, once the bias is removed, $\widehat{\rho}_{\mathrm{UB}}$ can be negative or zero. To deal with this problem, we define some $\rho_{\min }$ and clip the estimate to this value if it is lower.

\section{AnAlytical Performance}

Since the asymptotic ML performance is described by the $\mathrm{CRB}$, here we focus on the exact performance of the DA estimator. At high SNR, this analysis holds for the NDA estimator, as well. Performance is evaluated both when an assumed polynomial channel model order $L_{E}$ coincides with the true channel model order $L_{C}$, and when it does not, giving rise to model-order mismatch. The analysis is carried out for complex channels, but can easily be extended to real channels (by substituting $2 L_{E}$ with $L_{E}$ ).

\section{A. Correct Model-Order Performance}

As stated earlier, in the DA case, $\widehat{\rho}$ is a scaled noncentral $F$ distributed random variable. The variance of $x \sim \mathcal{F}_{\nu_{1}, \nu_{2}}(\lambda)$ is [17]

$$
\operatorname{VAR}=2\left(\frac{\nu_{2}}{\nu_{1}}\right)^{2} \frac{\left(\nu_{1}+\lambda\right)^{2}+\left(\nu_{1}+2 \lambda\right)\left(\nu_{2}-2\right)}{\left(\nu_{2}-2\right)^{2}\left(\nu_{2}-4\right)} .
$$

Thus, the mean-square error (MSE) of $\widehat{\rho}_{\mathrm{UB}}$ is directly obtained as

$$
\mathrm{MSE}=\frac{(2 L+2 N \rho)^{2}+(2 L+4 N \rho)(2 N-2 L-2)}{2 N^{2}(2 N-2 L-4)} .
$$

It is easy to verify that asymptotically, i.e., as long as $N \gg L$, the MSE attains the CRB of (7) as expected

$$
\operatorname{MSE} \stackrel{N \gg L}{\longrightarrow} \frac{2 \rho+\rho^{2}}{N}=\operatorname{CRB}_{\mathrm{DA}}(\rho)
$$

Examining the derivative of the MSE with respect to $L$

$$
\frac{\partial \mathrm{MSE}}{\partial L}=\frac{\left(\rho^{2}+2 \rho+1\right) N^{2}-(3+2 \rho) N+2}{N^{2}(2 N-2 L-4)^{2}}>0
$$

reveals that the derivative is positive for all $N>3$, and therefore, increasing $L$ increases the MSE. However, as previously stated, asymptotically, this increase is negligible

$$
\frac{\partial \mathrm{MSE}}{\partial L} \stackrel{N \gg L}{\longrightarrow} \frac{\rho^{2}+2 \rho+1}{4 N^{2}} \rightarrow 0 .
$$

In addition, as expected, the MSE in complex channels $(2 L)$ is higher than the MSE in real channel $(L)$. Again, asymptotically, this increase is negligible.

\section{B. Mismatched Model-Order Performance}

Performance analysis under channel model-order mismatch will give insight as to which $L_{E}$ should be selected when deriving an estimator for an actual time-varying channel.

When the estimator is based on a higher model order than the true model order $\left(L_{E}>L_{C}\right)$, the estimator is still a scaled noncentral $F$ random variable, only with a different scale factor and different number of DOFs. As long as $L_{E} \ll N$, the MSE does not increase, due to using a higher $L_{E}$ estimator than is necessary. The more interesting question is how the estimator behaves when the selected estimator model order is too low $\left(L_{E}<L_{C}\right)$. In an $L_{C}$ model-order channel, the $L_{E}$ model-order estimator is a scaled, doubly noncentral $F$ random variable [18]

$$
\begin{aligned}
\frac{N-L_{E}}{L_{E}} \widehat{\rho} & \sim \mathcal{F}_{2 L_{E}, 2 N-2 L_{E}}(2 N \eta, 2 N \rho-2 N \eta) \\
\eta & =\frac{\mathbf{c}_{L_{C}}^{H} \mathbf{T}_{L_{C}}^{T} \mathbf{T}_{L_{E}}\left(\mathbf{T}_{L_{E}}^{T} \mathbf{T}_{L_{E}}\right)^{-1} \mathbf{T}_{L_{E}}^{T} \mathbf{T}_{L_{C}} \mathbf{c}_{L_{C}}}{N \sigma^{2}}
\end{aligned}
$$

where $\mathcal{F}_{\nu_{1}, \nu_{2}}\left(\lambda_{1}, \lambda_{2}\right)$ denotes a doubly noncentral $F$ distribution with DOFs $\nu_{1}$ and $\nu_{2}$, and with noncentrality parameters $\lambda_{1}$ and $\lambda_{2}$. Estimator performance is determined by the moments of a doubly noncentral $z \sim \mathcal{F}_{\nu_{1}, \nu_{2}}\left(\lambda_{1}, \lambda_{2}\right)$ random variable which are (see Appendix III)

$$
\begin{aligned}
\mathrm{E}[z]= & \left(\frac{\nu_{2}}{\nu_{1}}\right)\left[\nu_{1}+\lambda_{1}\right] \frac{e^{-\frac{\lambda_{2}}{2}}}{2} \frac{\Gamma\left(\frac{\nu_{2}}{2}-1\right)}{\Gamma\left(\frac{\nu_{2}}{2}\right)} F\left(\frac{\nu_{2}}{2}-1, \frac{\nu_{2}}{2}, \frac{\lambda_{2}}{2}\right) \\
\mathrm{E}\left[z^{2}\right]= & \left(\frac{\nu_{2}}{\nu_{1}}\right)^{2}\left[2 \nu_{1}+4 \lambda_{1}+\left(\nu_{1}+\lambda_{1}\right)^{2}\right] \\
& \cdot \frac{e^{-\frac{\lambda_{2}}{2}}}{4} \frac{\Gamma\left(\frac{\nu_{2}}{2}-2\right)}{\Gamma\left(\frac{\nu}{2}\right)} F\left(\frac{\nu_{2}}{2}-2, \frac{\nu_{2}}{2}, \frac{\lambda_{2}}{2}\right)
\end{aligned}
$$

where $\Gamma(\cdot)$ is the Gamma function, and $F(\cdot, \cdot, \cdot)$ is the hypergeometric function [19].

For example, let us examine the performance of the $L_{E}=1$ order estimator in the $L_{C}=2$ order channel. For simplicity, we 


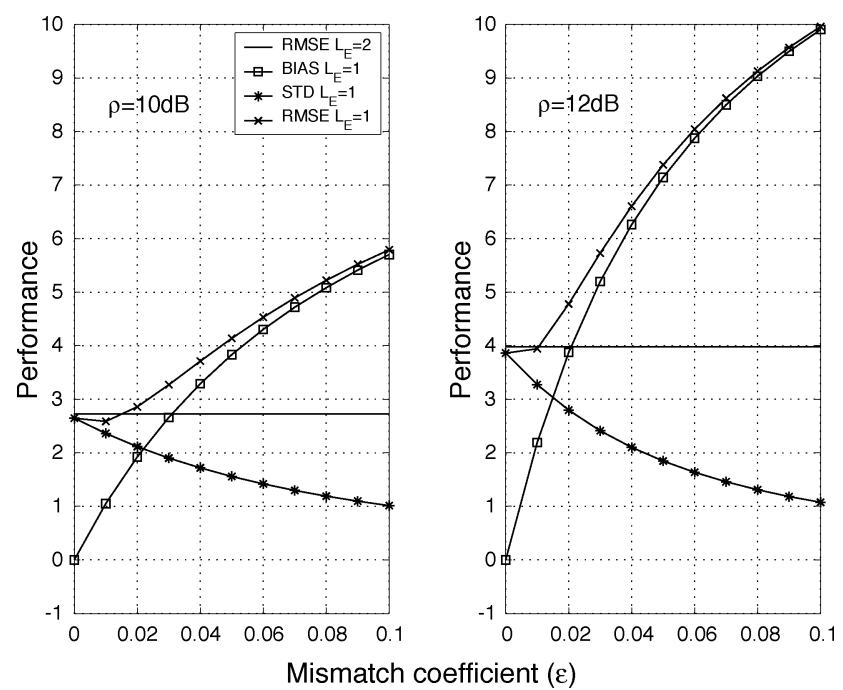

Fig. 2. DA performance of $\widehat{\rho}_{1}\left(L_{E}=1\right.$ model order $)$ estimator in a mismatched $L_{C}=2$ model-order channel, and performance of $\hat{\rho}_{2}\left(L_{E}=2\right)$, $\mathbf{T}^{T} \mathbf{T}=N \mathbf{I}, c_{0}^{2}=(1-\epsilon) \rho$, and $c_{1}^{2}=\epsilon \rho$, for $\rho^{\mathrm{dB}}=10 \mathrm{~dB}$ and $\rho^{\mathrm{dB}}=12 \mathrm{~dB}$.

choose $\left\{t_{1} \cdots t_{N}\right\}$ such that $\mathbf{T}^{T} \mathbf{T}=N \mathbf{I}$. In addition, we define $\sigma^{2}=1, c_{0}^{2}=(1-\epsilon) \rho$, and $c_{1}^{2}=\epsilon \rho$, where $0 \leq \epsilon \leq 1$ is a mismatch parameter which divides the energy between the two coefficients. When $\epsilon=0$, there is no model mismatch. Under these assumptions, $\eta=(1-\epsilon) \rho$ in (25). For comparison, the performance of the $L_{E}=2$ estimator, based on the correct model order, does not depend on $\epsilon$.

Plotting the theoretical bias, standard deviation (STD) and root mean-squared error (RMSE) of these estimators (Fig. 2) reveals the following properties. The use of a mismatched estimator usually degrades the performance. The model-order error causes a severe bias, and although there is a decrease in variance, the total MSE increases. This performance degradation increases as the mismatch parameter $\epsilon$ gets larger. As dramatically seen in Fig. 2, when the SNR increases, the mismatch effect is more significant. This is expected, since when the SNR increases, the model error dominates the error caused by noise. When the mismatch parameter is very low, and, in particular, when $\epsilon=0$, the $L_{E}=1$ model-order estimator becomes the correct estimator, and therefore, is slightly better than the $L_{E}=2$ model-order estimator. As proved in the previous section, if $N \gg L_{E}$, this difference is negligible.

\section{EMPIRICAL PERFORMANCE}

In this section, we evaluate the estimators' performance using computer simulation. First, we examine the performance of the various NDA estimators in constant channels. Fig. 3 shows the empirical RMSE of the estimators when compared with the CRB. It is seen that, as expected, the DD estimator offers acceptable performance only for sufficiently high SNRs (where the decisions on the symbols are highly reliable), eventually reaching the CRB. (Note that DD can only be used in real channels.) On the other hand, while the MM estimator performance is better than that of DD at low SNRs, high-SNR performance is inferior. Last, the newly proposed estimators (with $L_{E}=1,2,5$ EM iterations) are seen to outperform both DD and MM, yielding statistically efficient estimation over a wide range of SNRs.
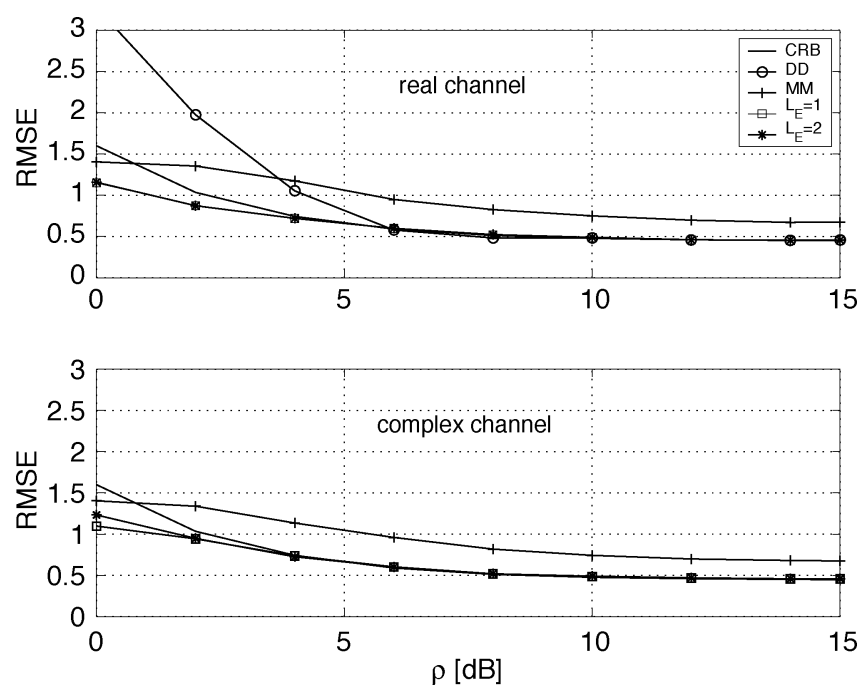

Fig. 3. NDA RMSE of $\widehat{\rho}^{\mathrm{dB}}$, QPSK constellation, $N=100$, and $L_{c}=1$.
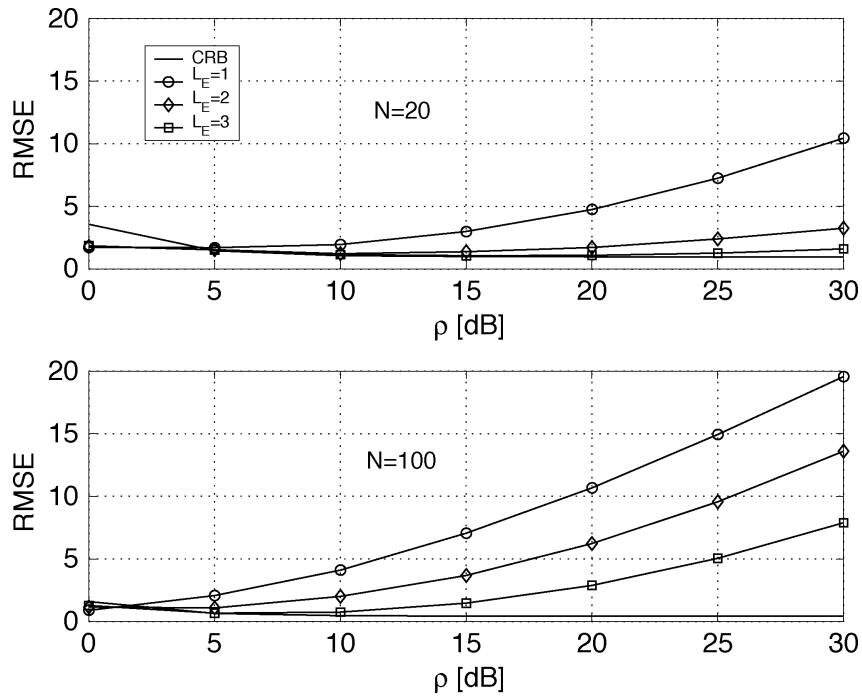

Fig. 4. NDA normalized RMSE of $\widehat{\rho}^{\mathrm{dB}}$, real Clarke's time-varying channel, QPSK constellation, $f_{s}=24300 \mathrm{~Hz}$, and $f_{d}=100$.

The low SNR RMSE of the estimators is smaller than the CRB due to a severe bias observed in this region. As predicted by ML properties, using a higher number of symbols eliminates this behavior. It is also seen that the degradation in performance due to overestimating the channel's model order (i.e., using $L_{E}=2$ in a constant channel) is negligible.

Next, we examine the estimators' performance in Clarke's time-varying channel model. In the simulations, we generate random channels according to Clarke's model, but normalized them so they will fit specific deterministic SNRs. This enables us to compare the performance with the previously derived CRB at a particular SNR. Fig. 4 reveals that in the low-SNR region, the main cause for performance degradation is the NDA nature of the problem. Here, the model error is negligible compared with the noise error. Therefore, the results are similar to those presented in Fig. 3. On the other hand, in the high-SNR regions, the unknown symbols are easily estimated, and the main cause for performance degradation stems from the inaccurate channel 
model. A significant difference in performance among the estimators is observed. As expected, the higher order estimators perform better, especially as $\left(f_{d} / f_{s}\right) N$ increases. This is particularly true, at high SNR, when the model error dominates the noise errors and introduces a severe bias. It can be inferred that for a given SNR, the CRB can be asymptotically attained with increasing model order.

\section{CONCLUSIONS}

This paper has considered the problem of DA and NDA SNR estimation in time-varying fading channels. The fading channel has been modeled as a polynomial-in-time. The associated CRB and the ML estimator have been derived, and the EM algorithm was used for implementing the ML estimator in NDA scenarios. The performance of the DA estimators was analytically computed with and without channel polynomial order mismatch. Simulation results have been presented to investigate estimator performance for fading channels, with temporal correlation governed by the well-known Clarke model. Theoretical analysis has shown that the accuracy of SNR estimation should not degrade due to the channel's time variation. It was found that the new estimators yield dramatic performance improvements over the conventional constant channel estimator at a moderate increase in computational complexity. Statistical efficiency is observed over a wide range of SNRs and number of measurements.

\section{APPENDIX I \\ NDA FIM ELEMENT DERIVATION FOR REAL AND COMPLEX CHANNELS}

Derivation of the elements of the FIM follow from tedious but straightforward derivations paralleling those in [9]. Due to space limitations, we now provide the final FIM elements for both the real and complex polynomial-in-time channel. More details can be found in [20].

Due to the independent received samples, the overall FIM can be calculated as the sum of $N$ samples' FIMs

$$
\mathbf{J}_{\boldsymbol{\theta} \boldsymbol{\theta}^{T}}=\sum_{n=1}^{N} \mathbf{J}_{\boldsymbol{\theta} \boldsymbol{\theta}^{T}}^{(n)}, \quad \mathbf{J}_{\boldsymbol{\theta} \boldsymbol{\theta}^{T}}^{(n)}=\mathrm{E}\left[-\frac{\partial^{2} \log p\left(y_{n} ; \theta\right)}{\partial \boldsymbol{\theta} \partial \boldsymbol{\theta}^{T}}\right] .
$$

The $n$th FIM, $\mathbf{J}_{\boldsymbol{\theta} \boldsymbol{\theta}^{T}}^{(n)}$, for estimating $\boldsymbol{\theta}=\left[\mathbf{c}^{T}, \sigma^{2}\right]$ in a real channel, is a $\left(L_{C}+1\right) \times\left(L_{C}+1\right)$ matrix with the following elements:

$$
\begin{aligned}
& \mathbf{J}_{\mathbf{c}_{i} \mathbf{c}_{k}}^{(n)}=t_{n}^{i+k} \frac{2}{\sigma^{2}} f\left(\rho_{n}\right) \quad \mathbf{J}_{\sigma^{2} \mathbf{c}_{i}}^{(n)}=t_{n}^{i} \frac{2 h_{n}}{\sigma^{4}}\left[1-f\left(\rho_{n}\right)\right] \\
& \mathrm{J}_{\sigma^{2} \sigma^{2}}^{(n)}=\left[\frac{1}{\sigma^{4}}-\frac{2 h_{n}^{2}\left[1-f\left(\rho_{n}\right)\right]}{\sigma^{6}}\right]
\end{aligned}
$$

where $\rho_{n}=2\left(\mathbf{c}^{H} \mathbf{t}_{n} \mathbf{t}_{n}^{T} \mathbf{c} / \sigma^{2}\right)$ in BPSK constellations, and $\rho_{n}=$ $\left(\mathbf{c}^{H} \mathbf{t}_{n} \mathbf{t}_{n}^{T} \mathbf{c} / \sigma^{2}\right)$ in QPSK constellations, and where $f\left(\rho_{n}\right)$ is one for the DA scenario and as in (9) for the NDA scenario.

The FIM for estimating $\boldsymbol{\theta}=\left[\boldsymbol{\alpha}^{T}, \boldsymbol{\beta}^{T}, \sigma^{2}\right]^{T}$ in a complex channel is a $\left(2 L_{C}+1\right) \times\left(2 L_{C}+1\right)$ matrix with the following elements:

$$
\begin{gathered}
\mathbf{J}_{\alpha_{i} \alpha_{k}}^{(n)}=t_{n}^{i+k}\left[\frac{2 R_{n}^{2}}{\sigma^{2}\left|h_{n}\right|^{2}} f\left(\rho_{n}\right)+\frac{2 I_{n}^{2}}{\sigma^{2}\left|h_{n}\right|^{2}} g\left(\rho_{n}\right)\right] \\
\mathbf{J}_{\beta_{i} \beta_{k}}^{(n)}=t_{n}^{i+k}\left[\frac{2 I_{n}^{2}}{\sigma^{2}\left|h_{n}\right|^{2}} f\left(\rho_{n}\right)+\frac{2 R_{n}^{2}}{\sigma^{2}\left|h_{n}\right|^{2}} g\left(\rho_{n}\right)\right]
\end{gathered}
$$

$\mathbf{J}_{\alpha_{i} \beta_{k}}^{(n)}=t_{n}^{i+k}\left[\frac{2 R_{n} I_{n}}{\sigma^{2}\left|h_{n}\right|^{2}} f\left(\rho_{n}\right)-\frac{2 R_{n} I_{n}}{\sigma^{2}\left|h_{n}\right|^{2}} g\left(\rho_{n}\right)\right]$

$\mathbf{J}_{\sigma^{2} \alpha_{i}}^{(n)}=t_{n}^{i} \frac{2 R_{n}}{\sigma^{4}}\left[1-f\left(\rho_{n}\right)\right], \quad \mathbf{J}_{\sigma^{2} \beta_{i}}^{(n)}=t_{n}^{i} \frac{2 I_{n}}{\sigma^{4}}\left[1-f\left(\rho_{n}\right)\right]$

$\mathbf{J}_{\sigma^{2} \sigma^{2}}^{(n)}=\frac{1-2 \rho_{n}\left[1-f\left(\rho_{n}\right)\right]}{\sigma^{4}}$

where $R_{n}=\Re\left\{h_{n}\right\}, I_{n}=\Im\left\{h_{n}\right\}$, and where $g\left(\rho_{n}\right)$ is zero in the DA scenario, and as follows in the NDA scenario:

$$
g\left(\rho_{n}\right)=1-\frac{e^{-\frac{\rho_{n}}{2}}}{\sqrt{2 \pi}} \int_{-\infty}^{\infty} \frac{e^{-\frac{1}{2} z^{2}}}{\cosh \left(\sqrt{\rho_{n}} z\right)} d z .
$$

\section{APPENDIX II}

THE FunCTION $f(\rho)$

In this appendix, we prove the convergence of the function

$$
f(\rho)=1-\frac{\mathrm{e}^{-\frac{\rho}{2}}}{\sqrt{2 \pi}} \int \frac{y^{2} \mathrm{e}^{-\frac{1}{2} y^{2}}}{\cosh (y \sqrt{\rho})} d y .
$$

The right-hand side integral is positive, and therefore, $f(\rho) \leq 1$. On the other hand, using $\cosh x \geq(1 / 2) e^{|x|}$, a lower bound on $f(\rho)$ can be obtained

$$
\begin{aligned}
f(\rho) & =1-\frac{\mathrm{e}^{-\frac{\rho}{2}}}{\sqrt{2 \pi}} \int_{-\infty}^{\infty} \frac{y^{2} \mathrm{e}^{-\frac{1}{2} y^{2}}}{\cosh (y \sqrt{\rho})} d y \\
& \geq 1-e^{-\frac{\rho}{2}} \frac{1}{\sqrt{2 \pi}} \int_{-\infty}^{\infty} y^{2} \mathrm{e}^{-\frac{1}{2} y^{2}} 2 \mathrm{e}^{-(|y| \sqrt{\rho})} d y \stackrel{\rho \gg 1}{\longrightarrow} 1 .
\end{aligned}
$$

This last integral has a closed form, involving the error function, e.g., [19], which is known to converge to one. Thus, at high SNR, the function $f(\rho)$ is bounded by one from both sides and converges to it.

\section{APPENDIX III}

\section{MOMENTS OF A DOUbly NONCENTRAL $F$ RANDOM VARIABLE}

In this appendix, we find the moments of a doubly noncentral $F$ distributed random variable [18]: $f \sim \mathcal{F}_{\nu_{1}, \nu_{2}}\left(\lambda_{1}, \lambda_{2}\right)$, where $\nu_{1}$ and $\nu_{2}$ are the DOFs, and $\lambda_{1}$ and $\lambda_{2}$ are the noncentrality parameters. This random variable is known to be the ratio of two independent noncentral chi-squared random variables $f=$ $\left(x_{1} / \nu_{1}\right) /\left(x_{2} / \nu_{2}\right)$, where $x_{i} \sim \chi_{\nu_{i}}^{2}\left(\lambda_{i}\right)$ denotes a noncentral chi-squared random variable with noncentrality parameter $\lambda_{i}$ and $\nu_{i}$ DOFs. Therefore, the moments of $f$ are

$$
\mathrm{E}\left[f^{h}\right]=\left(\frac{\nu_{2}}{\nu_{1}}\right)^{h} \mathrm{E}\left[\left(\frac{x_{1}}{x_{2}}\right)^{h}\right]=\left(\frac{\nu_{2}}{\nu_{1}}\right)^{h} \mathrm{E}\left[x_{1}^{h}\right] \mathrm{E}\left[\frac{1}{x_{2}^{h}}\right]
$$

where the last equality results from the independence of $x_{1}$ and $x_{2}$. The positive moments of the numerator are, e.g., [21]

$$
\mathrm{E}\left[x_{1}\right]=\nu_{1}+\lambda_{1}, \quad \mathrm{E}\left[x_{1}^{2}\right]=2 \nu_{1}+4 \lambda_{1}+\left(\nu_{1}+\lambda_{1}\right)^{2} .
$$

The moments of the denominator are derived using the moment-generating function, e.g., [17]: $M_{2}(z)=\mathrm{E}\left[\mathrm{e}^{z x_{2}}\right]=(1-$ $2 z)^{-\nu_{2} / 2} e^{\left(\left(z \lambda_{2}\right) /(1-2 z)\right)}$. Introducing the Gamma integral, e.g., 
[19]: $\Gamma(h)=\int_{0}^{\infty} \widetilde{z}^{h-1} e^{-\tilde{z}} d \widetilde{z}$, substituting $\widehat{z}=z_{2} z$, rearranging, and applying the expectation yields

$$
\begin{aligned}
\mathrm{E}\left[\frac{1}{x_{2}^{h}}\right] & =\frac{1}{\Gamma(h)} \int_{0}^{\infty} z^{h-1} M_{2}(-z) d z \\
& =\frac{1}{\Gamma(h)} \int_{0}^{\infty} z^{h-1}(1+2 z)^{-\frac{\nu_{2}}{2}} e^{-\frac{z \lambda_{2}}{1+2 z}} d z .
\end{aligned}
$$

Substituting $x=1+2 z$

$$
\begin{aligned}
\mathrm{E}\left[\frac{1}{x_{2}^{h}}\right] & =\frac{1}{\Gamma(h)} \frac{1}{2} \int_{1}^{\infty}\left(\frac{x-1}{2}\right)^{h-1} x^{-\frac{\nu_{2}}{2}} e^{-\frac{\lambda_{2}(x-1)}{2 x}} d x \\
& =\frac{1}{2^{h}} e^{-\frac{\lambda_{2}}{2}} \frac{\Gamma\left(\frac{\nu}{2}-h\right)}{\Gamma\left(\frac{\nu}{2}\right)} F\left(\frac{\nu}{2}-h, \frac{\nu}{2}, \frac{\lambda_{2}}{2}\right)
\end{aligned}
$$

where $F(\cdot, \cdot, \cdot)$ is the hypergeometric function, e.g., [22]. Using these expectations in (33) provides a closed form of the moments of the doubly noncentral $F$ distribution.

\section{REFERENCES}

[1] K. Balachandran, S. R. Kadaba, and S. Nanda, "Channel quality estimation and rate adaption for cellular mobile radio," IEEE J. Sel. Areas Commun., vol. 17, no. 7, pp. 1244-1256, Jul. 1999.

[2] T. A. Summers and S. G. Wilson, "SNR mismatch and online estimation in turbo decoding," IEEE Trans. Commun., vol. 46, no. 4, pp. 421-423, Apr. 1998.

[3] R. B. Kerr, "On signal and noise level estimation in a coherent PCM channel," IEEE Trans. Aerosp. Electron. Syst., vol. AES-2, no. 3, pp. 450-454, Jul. 1966.

[4] R. M. Gagliardi and C. M. Thomas, "PCM data reliability monitoring through estimation of signal-to-noise ratio," IEEE Trans. Commun., vol. COM-16, no. 6, pp. 479-486, Jun. 1968.

[5] D. R. Pauluzzi and N. C. Beaulieu, "A comparison of SNR estimation techniques for the AWGN channel," IEEE Trans. Commun., vol. 48, no. 10 , pp. 1681-1691, Oct. 2000.

[6] N. C. Beaulieu, A. S. Toms, and D. R. Pauluzzi, "Comparison of four SNR estimators for QPSK modulations," IEEE Commun. Lett., vol. 4, no. 2, pp. 43-45, Feb. 2000.

[7] M. Turkboylari and G. L. Stuber, "An efficient algorithm for estimating the signal-to-interference ratio in TDMA cellular systems," IEEE Trans. Commun., vol. 46, no. 6, pp. 728-731, Jun. 1998.

[8] M. Andersin, N. B. Mandayam, and R. D. Yates, "Subspace-based estimation of the signal-to-interference ratio for TDMA cellular systems," in Proc. IEEE 46th Veh. Technol. Conf., Apr. 1996, pp. 742-748.

[9] N. S. Alagha, "Cramer-Rao bounds of SNR estimates for BPSK and QPSK modulated signals," IEEE Commun. Lett., vol. 5, no. 1, pp. 10-12, Jan. 2001.

[10] S. M. Kay, Fundamentals of Statistical Signal Processing - Estimation Theory. Englewood Cliffs, NJ: Prentice-Hall, 1993.

[11] C. Anton-Haro, J. A. R. Fonollosa, C. Fauli, and J. R. Fonollosa, "On the inclusion of channel's time dependence in a hidden Markov model for blind channel estimation," IEEE Trans. Veh. Technol., vol. 50, no. 3, pp. 867-873, May 2001.

[12] G. L. Stuber, Principles of Mobile Communication. Norwell, MA: Kluwer, 1996.

[13] D. K. Borah and B. D. Hart, "A robust receiver structure for time-varying, frequency-flat, Rayleigh fading channels," IEEE Trans. Commun., vol. 47, no. 3, pp. 360-364, Mar. 1999.

[14] E. Serpedin, G. B. G. P. Ciblat, and P. Loubaton, "Performance analysis of blind carrier phase estimators for general QAM constellations," IEEE Trans. Signal Process., vol. 49, no. 8, pp. 1816-1823, Aug. 2001.
[15] A. Wiesel, J. Goldberg, and H. Messer, "Non-data-aided signal-tonoise-ratio estimation," in Proc. IEEE Int. Conf. Commun., Apr. 2002, vol. 1, pp. 197-201.

[16] S. M. Kay, Fundamentals of Statistical Signal Processing-Detection Theory. Englewood Cliffs, NJ: Prentice-Hall, 1998.

[17] N. L. Johnson and S. Kotz, Distributions in Statistics-Continuous Univariate Distributions-2. Boston, MA: Houghton Mifflin, 1970.

[18] R. Price, "Some noncentral $F$ distributions expressed in closed form," Biometrika, vol. 51, no. 1-2, pp. 107-122, 1964.

[19] M. R. Spiegel, Mathematical Handbook of Formulas and Tables. New York: McGraw-Hill, 1990.

[20] A. Wiesel, "Signal-to-noise-ratio estimation in time-selective fading channels," M.Sc. thesis, Dept. Elect. Eng.-Syst., Tel Aviv Univ., Tel Aviv, Israel, 2002.

[21] A. M. Mathai and S. B. Provost, Quadratic Forms in Random Variables-Theory and Applications. New York: Marcel Dekker, 1992.

[22] G. Ryzhik, Table of Integrals, Series, and Products. New York: Academic, 1980.

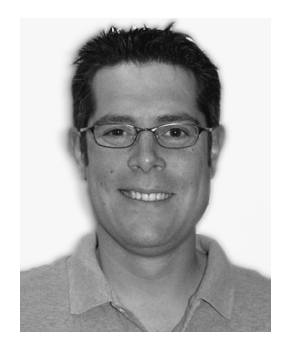

Ami Wiesel (S'02) received the B.Sc. degree in 2000 and the M.Sc. degree in 2002, both in electrical engineering and both from Tel-Aviv University, Tel-Aviv, Israel. He is currently working toward the Ph.D. degree with the Department of Electrical Engineering, Technion-Israel Institute of Technology, Haifa, Israel.

From June 2002 to March 2003, he was with the Department of Signal Theory and Communications, Universitat Politecnica de Catalunya, Barcelona, Spain. His main areas of interests include signal processing algorithms, convex optimization methods, and estimation theory.

Mr. Wiesel was awarded the Weinstein Study Prize in 2002 and the Intel Award in 2005. He received the Student Paper Award at SPAWC 2005, and was appointed a Viterbi Graduate Fellow for the winter of 2005-2006.

Jason Goldberg (SM'99) received the B.S.E.E. and Ph.D. degrees in electrical engineering from Worcester Polytechnic Institute, Worcester, MA, and University of London, London, U.K., in 1988 and 1993, respectively.

From 1993 to 1996, he was a Postdoctoral Researcher with the Polytechnic University of Catalonia, Barcelona, Spain. From 1996 to 2003, he was a Staff Researcher with Tel-Aviv University, Tel-Aviv, Israel, in the area of statistical and array signal processing. He was also a member of the Research Group, Wireless Computing and Communications Division, Intel Corp., Petach Tikva, Israel, from 1999 to 2003. Since 2004, he has worked on the design and analysis of baseband signal processing algorithms for GPS receivers with Global Locate, Inc., San Jose, CA.

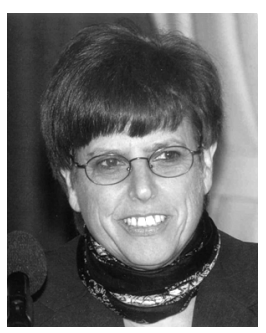

Hagit Messer-Yaron (F'01) received the Ph.D. degree in electrical engineering from Tel-Aviv University, Tel-Aviv, Israel.

After a one-year post-doctoral appointment at Yale University, New Haven, CT, she joined the faculty of Engineering at Tel-Aviv University in 1986, where she is currently a Professor of Electrical Engineering. From February 2000 to March 2003, she was the Chief Scientist and the Head of the Directorate of Science at the Ministry of Science, Israel. Since July 2004, she has also been the Head of the Porter School for Environmental Studies at Tel-Aviv University. She is an expert in statistical signal processing and applications to source localization, communication, and radar systems. She has published about 60 refereed journal papers, over 100 papers in conference proceedings, and she has supervised over 50 graduate students, all in the field of signal processing and statistical signal analysis. She has held international visiting positions and has been involved in the organization of international conferences and workshops.

Dr. Hagit Messer-Yaron is a member of Technical Committees of the IEEE Signal Processing Society since 1993. She has been an Associate Editor of the IEEE Transactions on Signal Processing and of the IEEE Signal PROCESSING LETTERS for parameter estimation, detection, and performance analysis. 